\title{
Analytical Approximate Solutions for the Cubic-Quintic Duffing Oscillator in Terms of Elementary Functions
}

\author{
A. Beléndez, ${ }_{y}^{1,2}$ M. L. Alvarez, ${ }^{1,2}$ J. Francés, ${ }^{1,2}$ S. Bleda, ${ }^{1,2}$ \\ T. Beléndez, ${ }^{1,2}$ A. Nájera, ${ }^{3}$ and E. Arribas ${ }^{4}$ \\ ${ }^{1}$ Departamento de Física, Ingeniería de Sistemas y Teoría de la Señal, Universidad de Alicante, \\ Apartado 99, 03080 Alicante, Spain \\ ${ }^{2}$ Instituto Universitario de Física Aplicada a las Ciencias y las Tecnologías, \\ Universidad de Alicante, Apartado 99, 03080 Alicante, Spain \\ ${ }^{3}$ Departamento de Ciencias Médicas, Facultad de Medicina, Universidad de Castilla-La Mancha, \\ C/Almansa No. 14, 02006 Albacete, Spain \\ ${ }^{4}$ Departamento de Física Aplicada, Escuela Superior de Ingeniería Informática, \\ Universidad de Castilla-La Mancha, Avenida de España s/n, 02071 Albacete, Spain
}

Correspondence should be addressed to A. Beléndez, a.belendez@ua.es

Received 28 June 2012; Accepted 13 August 2012

Academic Editor: Livija Cveticanin

Copyright (C) 2012 A. Beléndez et al. This is an open access article distributed under the Creative Commons Attribution License, which permits unrestricted use, distribution, and reproduction in any medium, provided the original work is properly cited.

Accurate approximate closed-form solutions for the cubic-quintic Duffing oscillator are obtained in terms of elementary functions. To do this, we use the previous results obtained using a cubication method in which the restoring force is expanded in Chebyshev polynomials and the original nonlinear differential equation is approximated by a cubic Duffing equation. Explicit approximate solutions are then expressed as a function of the complete elliptic integral of the first kind and the Jacobi elliptic function $\mathrm{cn}$. Then we obtain other approximate expressions for these solutions, which are expressed in terms of elementary functions. To do this, the relationship between the complete elliptic integral of the first kind and the arithmetic-geometric mean is used and the rational harmonic balance method is applied to obtain the periodic solution of the original nonlinear oscillator.

\section{Introduction}

The cubic-quintic Duffing equation is a differential equation with third- and/or fifth-power nonlinearity [1]. Due to the presence of this fifth-power nonlinearity added to the third nonlinearity of the common Duffing equation, this oscillator is difficult to handle. As Lai et al. pointed out [1], the cubic-quintic Duffing equation can be found in the modelling of different systems and we advise readers to consult this paper to learn more about these applications 
and a good set of references in which this strongly nonlinear equation can be found. Among these systems modelled by the cubic-quintic Duffing equation we would like to mention the nonlinear dynamics of a slender elastica, the compound $\mathrm{KdV}$ equation in nonlinear wave systems, or the propagation of a short electromagnetic pulse in a nonlinear medium [1-3].

The purpose of this paper is to present analytical approximations to the periodic solutions to the cubic-quintic Duffing oscillator [1-3] in terms of elementary functions. To do this, we use the results previously obtained [4] using the Chebyshev series expansion of the restoring force $[5,6]$ for a quintic Duffing oscillator. Using this approximation, the original nonlinear differential equation is replaced by a cubic Duffing equation, which can be exactly solved. The replacement of the original nonlinear equation by an "approximate cubic Duffing equation" allows us to obtain an approximate frequency-amplitude relation as a function of the complete elliptic integral of the first kind and the solution in terms of the Jacobi elliptic function cn [4]. Using these first approximate equations, closed-form expressions for the approximate frequency and the solution in terms of elementary functions are obtained using the arithmetic-geometric mean combined with the (second-order) rational harmonic balance method.

In relation to cubication methods, Bravo-Yuste [7] introduced a cubication procedure based on the harmonic balance method and Bravo-Yuste and Martín-Sánchez [8] also developed a weighted mean square method of cubication. Recently, Beléndez et al. $[4,9,10]$ and Elías-Zùñiga et al. [11] employed similar procedures based on the use of Chebyshev polynomials. In particular, Elías-Zùñiga et al. combined the cubication method with the equivalent nonlinearization method developed by Cai et al. [12].

In the early 1980's and 1990's, much effort was put on renormalization techniques and the use of the well-known arithmetic-geometric mean to analyze nonlinear oscillators and, in particular, the old famous simple pendulum [13]. Recently, and also analyzing the nonlinear pendulum, it has been shown that it is possible to obtain an approximate expression for the complete elliptic integral of the first kind by using a Taylor series expansion [14], which is similar to the second approximation derived using the arithmetic-geometric mean. Finally, the rational harmonic balance method (RHBM) [15] is more difficult to apply than the common harmonic balance method (HBM) for oscillators with complex nonlinearities. However, we will see that the RHBM is easy to apply to the cubic Duffing equation obtained when the Chebyshev polynomials expansion is taken into account. The RHBM presents the advantage versus the usual HBM that the RHBM includes approximations for all the harmonics contributing to the periodic solution [16].

\section{Solution Procedure Using a Chebyshev Polynomials Expansion}

A cubic-quintic Duffing oscillator is a conservative autonomous oscillatory system, which can be described by the following second-order differential equation with cubic-quintic nonlinearities [1]:

$$
\frac{d^{2} x}{d t^{2}}+\lambda_{1} x+\lambda_{3} x^{3}+\lambda_{5} x^{5}=0
$$

with initial conditions

$$
x(0)=A, \quad \frac{d x}{d t}(0)=0,
$$


where $\lambda_{1} x+\lambda_{3} x^{3}+\lambda_{5} x^{5}$ is an odd function; $x$ and $t$ are dimensionless displacement and time variables, respectively; $\lambda_{1}, \lambda_{3}$, and $\lambda_{5}$ are positive constant parameters [1]. The angular frequency of these oscillations is denoted by $\omega$, which is a function of the initial amplitude A.

The cubic-quintic Duffing equation is difficult to handle because of the presence of strong nonlinearity and it has no known closed-form solution. However, the quintic Duffing equation $\left(\lambda_{1}=1, \lambda_{3}=0\right.$, and $\lambda_{5}=\varepsilon \neq 0$ in (2.1)) has been recently solved approximately by means of a cubication procedure based on the expansion of the nonlinear restoring force in terms of the Chebyshev polynomials [4]. To apply this procedure, firstly a reduced variable $y=x / A$ is introduced in (2.1) and (2.2) and the nonlinear function $g(y)=\lambda_{1} y+\lambda_{3} A^{2} y^{3}+$ $\lambda_{5} A^{4} y^{5}$ is expanded in terms of Chebyshev polynomials of the first kind $T_{n}(y)$. Retaining only the first two terms in this expansion, we obtain

$$
\begin{aligned}
g(y) & =\sum_{n=0}^{\infty} b_{2 n+1} T_{2 n+1}(y) \approx \sum_{n=0}^{1} b_{2 n+1} T_{2 n+1}(y) \\
& =b_{1} T_{1}(y)+b_{3} T_{3}(y)=\left(b_{1}-3 b_{3}\right) y+4 b_{3} y^{3}=\alpha y+\beta y^{3}
\end{aligned}
$$

where

$$
\begin{gathered}
b_{2 n+1}=\frac{2}{\pi} \int_{-1}^{+1} \frac{1}{\sqrt{1-y^{2}}} g(y) T_{2 n+1}(y) d y, \\
b_{1}=\lambda_{1}+\frac{3}{4} \lambda_{3} A^{2}+\frac{5}{8} \lambda_{5} A^{4}, \quad b_{3}=\frac{1}{4} \lambda_{3} A^{2}+\frac{5}{16} \lambda_{5} A^{4} .
\end{gathered}
$$

Then the nonlinear differential equation in (2.1) can be then approximated by the (cubic-) Duffing equation

$$
\frac{d^{2} y}{d t^{2}}+\alpha(A) y+\beta(A) y^{3}=0
$$

where

$$
\alpha=\lambda_{1}-\frac{5}{16} \lambda_{5} A^{4}, \quad \beta=\lambda_{3} A^{2}+\frac{5}{4} \lambda_{5} A^{4} .
$$

Equation (2.5) can be exactly solved and its solution can be written in terms of the Jacobian elliptic function cn [15]. Then the approximate frequency and solution for the cubicquintic equation in (2.1) will be the exact frequency and solution for the cubic equation in (2.5). Taking into account the well-known expressions for the frequency and the solution for the Duffing oscillator in (2.5), we can easily obtain the following analytical approximate 
expressions for the frequency and solution for the cubic-quintic Duffing oscillator as follows:

$$
\begin{aligned}
& \omega_{a}(A)=\frac{\pi \sqrt{\lambda_{1}+\lambda_{3} A^{2}+(15 / 16) \lambda_{5} A^{4}}}{2 K\left(\left(8 \lambda_{3} A^{2}+10 \lambda_{5} A^{4}\right) /\left(16 \lambda_{1}+16 \lambda_{3} A^{2}+15 \lambda_{5} A^{4}\right)\right)} \\
& y(t)=\operatorname{cn}\left(t \sqrt{\lambda_{1}+\lambda_{3} A^{2}+\frac{15}{16} \lambda_{5} A^{4}} ; \frac{8 \lambda_{3} A^{2}+10 \lambda_{5} A^{4}}{16 \lambda_{1}+16 \lambda_{3} A^{2}+15 \lambda_{5} A^{4}}\right)
\end{aligned}
$$

where $K(m)$ is the complete elliptic integral of the first kind

$$
K(m)=\int_{0}^{\pi / 2} \frac{d \theta}{\sqrt{1-m \sin ^{2} \theta}} .
$$

\section{Closed-Form Expression for the Approximate Frequency in Terms of Elementary Functions Using the Arithmetic-Geometric Mean}

As we can see from (2.7), the approximate frequency for the cubic-quintic Duffing oscillator is expressed in terms of the complete elliptic integral of the first kind, $K(m)$. However, it is also possible to obtain a closed-form expression for the approximate frequency in terms of elementary functions. The complete elliptic integral of the first kind (2.9) cannot be expressed in terms of elementary functions, but can be numerically evaluated with high precision by a simple procedure based on the arithmetic-geometric mean. This is due to the fact that the arithmetic-geometric mean is the basis of Gauss' method for the calculation of elliptic integrals [16-18]. The arithmetic-geometric mean of two numbers $p$ and $q, M(p, q)$ can be expressed in closed-form in terms of the complete elliptic integral of the first kind as [16]

$$
M(p, q)=\frac{(p+q) \pi}{4 K((p-q) /(p+q))}
$$

which allows us to write $K(m)$ in (2.9) as follows [16]:

$$
K(m)=\frac{\pi}{2 M(1, \sqrt{1-m})} .
$$

The Legendre form of the arithmetic-geometric mean is given by [16]

$$
M(1, z)=\prod_{n=0}^{\infty} \frac{1}{2}\left(1+k_{n}\right)
$$


where $z \equiv k_{0}$ and $k_{n+1}=2 \sqrt{k_{n}} /\left(1+k_{n}\right)$. From (3.3) it is possible to approximately calculate the arithmetic-geometric mean by choosing a finite number of terms in this equation as follows:

$$
M(1, z) \approx \prod_{n=0}^{N} \frac{1}{2}\left(1+k_{n}\right)
$$

From (2.7), (3.2), and (3.4) we obtain the following expression for the approximate frequency of the cubic-quintic Duffing oscillator:

$$
\omega_{a}^{\mathrm{agm}}(A)=\frac{1}{16}\left[\left(16 \lambda_{1}+8 \lambda_{3} A^{2}+5 \lambda_{5} A^{4}\right)^{1 / 4}+\left(16 \lambda_{1}+16 \lambda_{3} A^{2}+15 \lambda_{5} A^{4}\right)^{1 / 4}\right]^{2},
$$

where only two terms $(N=1)$ have been considered in (3.4)

$$
\begin{aligned}
M(1, z) & \approx \prod_{n=0}^{1} \frac{1}{2}\left(1+k_{n}\right)=\frac{1}{4}\left(1+k_{0}\right)\left(1+k_{1}\right) \\
& =\frac{1}{4}\left(1+k_{0}\right)\left(1+\frac{2 \sqrt{k_{0}}}{1+k_{0}}\right)=\frac{1}{4}(1+z+2 \sqrt{z})=\frac{1}{4}(1+\sqrt{z})^{2},
\end{aligned}
$$

where $z \equiv k_{0}=\sqrt{1-m}$ and $m=\left(8 \lambda_{3} A^{2}+10 \lambda_{5} A^{4}\right) /\left(16 \lambda_{1}+16 \lambda_{3} A^{2}+15 \lambda_{5} A^{4}\right)$ (2.7). The maximum value of $m$ reached for the cubic-quintic Duffing oscillator is $\lim _{\lambda_{5} A^{4} \rightarrow \infty} m=$ $10 / 15<2 / 3$ because for the case in which $\lambda_{3} \neq 0$ and $\lambda_{5}=0$ we obtain $\lim _{\lambda_{3} A^{2} \rightarrow \infty} m=8 / 16=$ $1 / 2<2 / 3$. We can see that (3.5) is a very simple and easily computable expression.

From (3.2) and (3.6) it can be seen that it is possible to take into account the following approximation for the complete elliptic integral of the first kind:

$$
K_{a}(m)=\frac{2 \pi}{\left[1+(1-m)^{1 / 4}\right]^{2}} .
$$

In Table 1 we present the percentage errors for comparison of the approximate expression for the complete elliptic integral of the first kind, $K_{a}(m)(3.7)$, with the exact one, $K(m)$, for the interval of values of $m$ corresponding to the cubic-quintic Duffing oscillator $(0 \leq m \leq 2 / 3)$. As we can see from this table, $K_{a}(m)$ is a very good approximation for $K(m)$ and the relative error is less than $0.087 \%$ for $m \leq 2 / 3$.

To verify if it is sufficient to consider only a two-term approximation in the Legendre form of the arithmetic-geometric mean, we compare $\omega_{a}(A)(2.7)$ and $\omega_{a}^{\text {agm }}(A)(3.5)$ for $\lambda_{5} \neq 0$ and for $\lambda_{3} \neq 0$ and $\lambda_{5}=0$. 
Table 1: Percentage errors for comparison of the approximate expression for the complete elliptic integral of the first kind, $K_{a}(m)(3.7)$, with the exact one, $K(m)$.

\begin{tabular}{lccc}
\hline$m$ & $K_{a}(m)$ & $K(m)$ & $(\%$ Error $)$ \\
\hline 0 & 1.570796 & 1.570796 & 0 \\
0.1 & 1.612441 & 1.612441 & 0.00000075 \\
0.2 & 1.659623 & 1.659624 & 0.000015 \\
0.3 & 1.713888 & 1.713889 & 0.00010 \\
0.4 & 1.777512 & 1.777519 & 0.00041 \\
0.5 & 1.854049 & 1.854075 & 0.0014 \\
0.6 & 1.949485 & 1.949568 & 0.0042 \\
$2 / 3$ & 2.028783 & 2.028959 & 0.0087 \\
\hline
\end{tabular}

For $\lambda_{5} \neq 0$ we obtain the following power series expansions for these equations for very large values of the oscillation amplitude $A$

$$
\begin{gathered}
\omega_{a}(A) \approx \frac{\pi \sqrt{15 \lambda_{5}}}{8 K(2 / 3)} A^{2}=0.749605 \sqrt{\lambda_{5}} A^{2}, \\
\omega_{a}^{\mathrm{agm}}(A) \approx \frac{\left(1+3^{1 / 4}\right)^{2} \sqrt{5 \lambda_{5}}}{16} A^{2}=0.749670 \sqrt{\lambda_{5}} A^{2} .
\end{gathered}
$$

Furthermore, we have the following:

$$
\lim _{\lambda_{5} A^{4} \rightarrow 0} \frac{\omega_{a}^{\mathrm{agm}}(A)}{\omega_{a}(A)}=1, \quad \lim _{\lambda_{5} A^{4} \rightarrow \infty} \frac{\omega_{a}^{\mathrm{agm}}(A)}{\omega_{a}(A)}=1.000087
$$

The maximum relative error for the frequency using the arithmetic-geometric mean approximation in (3.5) instead of (2.7) is $0.0087 \%$, which allows us to conclude that (3.6) is a suitable approximation to (3.3).

For $\lambda_{3} \neq 0$ and $\lambda_{5}=0$ we obtain the following power series expansions for these equations for very large values of the oscillation amplitude $A$ :

$$
\begin{gathered}
\omega_{a}(A) \approx \frac{\sqrt{2 \pi} \Gamma(3 / 4) \sqrt{\lambda_{3}}}{\Gamma(1 / 4)} A=0.847213 \sqrt{\lambda_{3}} A, \\
\omega_{a}^{\mathrm{agm}}(\lambda) \approx \frac{\left(2+2^{1 / 2}+2^{7 / 4}\right)^{2} \sqrt{\lambda_{3}}}{8} A=0.847225 \sqrt{\lambda_{3}} A,
\end{gathered}
$$

where $\Gamma(z)$ is the Euler gamma function. Furthermore, we have the following equations:

$$
\lim _{\lambda_{3} A^{2} \rightarrow 0} \frac{\omega_{a}^{\mathrm{agm}}(A)}{\omega_{a}(A)}=1, \quad \lim _{\lambda_{3} A^{2} \rightarrow \infty} \frac{\omega_{a}^{\mathrm{agm}}(A)}{\omega_{a}(A)}=1.000014
$$

Now the maximum relative error for the frequency using the arithmetic-geometric mean approximation in (3.5) instead of (2.7) is as low as $0.0014 \%$, which allows us to conclude that (3.6) is a suitable approximation to (3.3). 


\section{Closed-Form Expression for the Solution in Terms of Elementary Functions Using the Rational Harmonic Balance Method}

In order to obtain an expression for the approximate solution in terms of elementary functions we consider that the approximate frequency is given by (3.5) and we apply the rational harmonic balance method (RHBM). By defining a new independent variable replacing the time variable, $\tau=\omega_{a}^{\mathrm{agm}} t,(2.5)$ can be expressed as follows:

$$
\omega^{2} \frac{d^{2} y}{d \tau^{2}}+\alpha y+\beta y^{3}=0
$$

where we have written $\omega=\omega_{a}^{\mathrm{agm}}$ for simplicity. We propose the following approximation to the periodic solution of (4.1)

$$
y(\tau)=\frac{(1-q) \cos \tau}{1-q \cos ^{2} \tau}, \quad 0 \leq|q|<1
$$

Note that this expression satisfies the prescribed initial conditions independently of parameter $q$, which is to be determined. It is easy to verify that substituting $q=-2 B_{2} /\left(1-B_{2}\right)$ in (4.2), we obtain the rational representation

$$
y(\tau)=\frac{\left(1+B_{2}\right) \cos \tau}{1+B_{2} \cos (2 \tau)}
$$

which is the expression usually considered in the bibliography for the second-order rational harmonic balance method [15]. However, (4.2) has the advantage that it allows us to simplify the procedure to obtain the coefficients of the Fourier series expansion of $y$ and $y^{3}$. These series can be written as follows:

$$
y^{\sigma}(\tau)=\sum_{n=1}^{\infty} c_{2 n+1}^{(\sigma)} \cos [(2 n+1) \tau], \quad \sigma=1,3
$$

where

$$
c_{2 n+1}^{(\sigma)}=\frac{4}{\pi} \int_{0}^{\pi / 2} y^{\sigma}(\tau) \cos [(2 n+1) \tau] d \tau .
$$

Substituting (4.4) into (4.1) the following result is obtained:

$$
\left(-\omega^{2} c_{1}^{(1)}+\alpha c_{1}^{(1)}+\beta c_{1}^{(3)}\right) \cos \tau+\mathrm{HOH}=0,
$$

where $\mathrm{HOH}$ stands for higher-order harmonics. Setting the coefficient of the lowest harmonic to zero gives

$$
\left(-\omega^{2}+\alpha\right) c_{1}^{(1)}+\beta c_{1}^{(3)}=0
$$


which can be solved to obtain $q$ as a function of $A$. Before doing this, it is necessary to obtain the values of the first Fourier coefficients $c_{1}^{(1)}$ and $c_{1}^{(3)}$ as a function of the unknown parameter $q$. To do this we firstly write the expression for $y^{\sigma}(\tau)$ as follows

$$
\begin{aligned}
y^{\sigma}(\tau) & =(1-q)^{\sigma} \cos ^{\sigma} \tau\left(1-q \cos ^{2} \tau\right)^{-\sigma}=(1-q)^{\sigma} \cos ^{\sigma} \tau \sum_{m=1}^{\infty}\left(\begin{array}{c}
-\sigma \\
m
\end{array}\right)(-1)^{m} q^{m} \cos ^{2 m} \tau \\
& =(1-q)^{\sigma} \sum_{m=0}^{\infty} \frac{(\sigma)_{m}}{m !} q^{m} \cos ^{2 m+\sigma} \tau=(1-q)^{\sigma} \sum_{m=0}^{\infty} \frac{\Gamma(\sigma+m)}{\Gamma(\sigma) \Gamma(m+1)} q^{m} \cos ^{2 m+\sigma} \tau
\end{aligned}
$$

and then

$$
\begin{gathered}
y(\tau)=(1-q) \sum_{m=0}^{\infty} q^{m} \cos ^{2 m+1} \tau \\
y^{3}(\tau)=\frac{1}{2}(1-q)^{3} \sum_{m=0}^{\infty}(m+1)(m+2) q^{m} \cos ^{2 m+3} \tau .
\end{gathered}
$$

Then Fourier coefficients $c_{1}^{(1)}$ and $c_{1}^{(3)}$ take the expressions

$$
\begin{aligned}
c_{1}^{(1)} & =\frac{4}{\pi} \int_{0}^{\pi / 2} y(\tau) \cos \tau d \tau=\frac{4}{\pi}(1-q) \sum_{m=0}^{\infty} q^{m} \int_{0}^{\pi / 2} \cos ^{2 m+2} \tau d \tau \\
& =\frac{4}{\pi}(1-q) \sum_{m=0}^{\infty} \frac{\sqrt{\pi} \Gamma(m+3 / 2) q^{m}}{2 \Gamma(m+2)}=\frac{2}{q}(\sqrt{1-q}-1+q), \\
c_{1}^{(3)} & =\frac{4}{\pi} \int_{0}^{\pi / 2} y^{3}(\tau) \cos \tau d \tau=\frac{2}{\pi}(1-q)^{3} \sum_{m=0}^{\infty}(m+1)(m+2) q^{m} \int_{0}^{\pi / 2} \cos ^{2 m+4} \tau d \tau \\
& =\frac{2}{\pi}(1-q)^{3} \sum_{m=0}^{\infty} \frac{\sqrt{\pi}(m+1)(m+2) \Gamma(m+5 / 2) q^{m}}{2 \Gamma(m+3)}=\frac{3}{4} \sqrt{1-q} .
\end{aligned}
$$

Substitution of (4.10) into (4.7), yields the following expression

$$
\frac{2}{q}\left(-\omega^{2}+\alpha\right)(\sqrt{1-q}-1+q)+\frac{3}{4} \beta \sqrt{1-q}=0
$$

Solving for $q$ gives

$$
q=-\frac{16}{9 \beta^{2}}\left[\left(\omega_{a}^{\mathrm{agm}}\right)^{2}-\alpha\right]\left[4\left(\omega_{a}^{\mathrm{agm}}\right)^{2}-4 \alpha-3 \beta\right]
$$

where $\alpha, \beta$ and $\omega_{a}^{\mathrm{agm}}$ are given in (2.6) and (3.5). From (4.12) we obtain for $\lambda_{5} \neq 0$

$$
\lim _{\lambda_{5} A^{4} \rightarrow \infty} q(A)=0.25072
$$




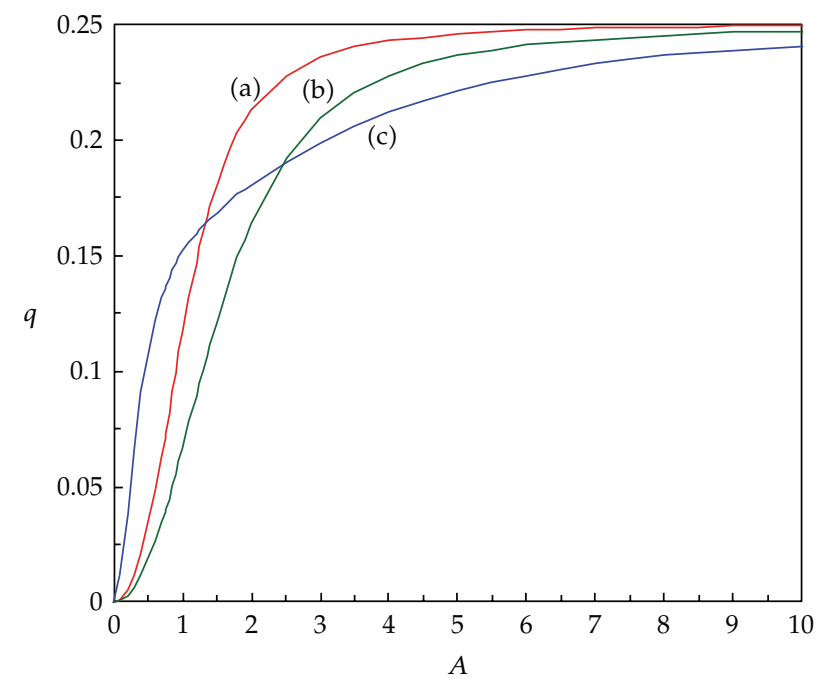

Figure 1: $q$ as a function of $A$ for cubic-quintic oscillators with the following parameters: (a) $\lambda_{1}=1, \lambda_{3}=1$, $\lambda_{5}=1$ (red), (b) $\lambda_{1}=5, \lambda_{3}=3, \lambda_{5}=1$ (green), and (c) $\lambda_{1}=1, \lambda_{3}=10, \lambda_{5}=1$ (blue).

and for $\lambda_{3} \neq 0$ and $\lambda_{5}=0$

$$
\lim _{\lambda_{3} A^{2} \rightarrow \infty} q(A)=0.16441
$$

In Figure 1 we have plotted parameter $q$ as a function of $A$ for different values of parameters $\lambda_{1}, \lambda_{3}$, and $\lambda_{5}$. Equations (4.13) and (4.14) allow us to know the maximum value of parameter qfor $\lambda_{5} \neq 0$ and $\lambda_{3} \neq 0\left(\lambda_{5}=0\right)$, respectively. In fact, for $\lambda_{3} \neq 0$ and $\lambda_{5}=0$ we have obtained an accurate approximate solution for the cubic Duffing equation.

\section{Comparison with the Exact Solution}

The exact frequency for the cubic-quintic Duffing oscillator can be obtained by direct integration of (2.1) using the initial conditions in (2.2). The result is (see Appendix B in [1])

$$
\omega_{e}(A)=\frac{\pi k_{1}}{2 \int_{0}^{\pi / 2}\left(1+k_{2} \sin ^{2} t+k_{3} \sin ^{4} t\right)^{-1 / 2} d t},
$$

where parameters $k_{1}, k_{2}$, and $k_{3}$ are given as follows:

$$
\begin{aligned}
& k_{1}=\sqrt{\lambda_{1}+\frac{1}{2} \lambda_{3} A^{2}+\frac{1}{3} \lambda_{5} A^{4}}, \\
& k_{2}=\frac{3 \lambda_{3} A^{2}+2 \lambda_{5} A^{4}}{6 \lambda_{1}+3 \lambda_{3} A^{2}+2 \lambda_{5} A^{4}} \\
& k_{3}=\frac{2 \lambda_{5} A^{4}}{6 \lambda_{1}+3 \lambda_{3} A^{2}+2 \lambda_{5} A^{4}} .
\end{aligned}
$$




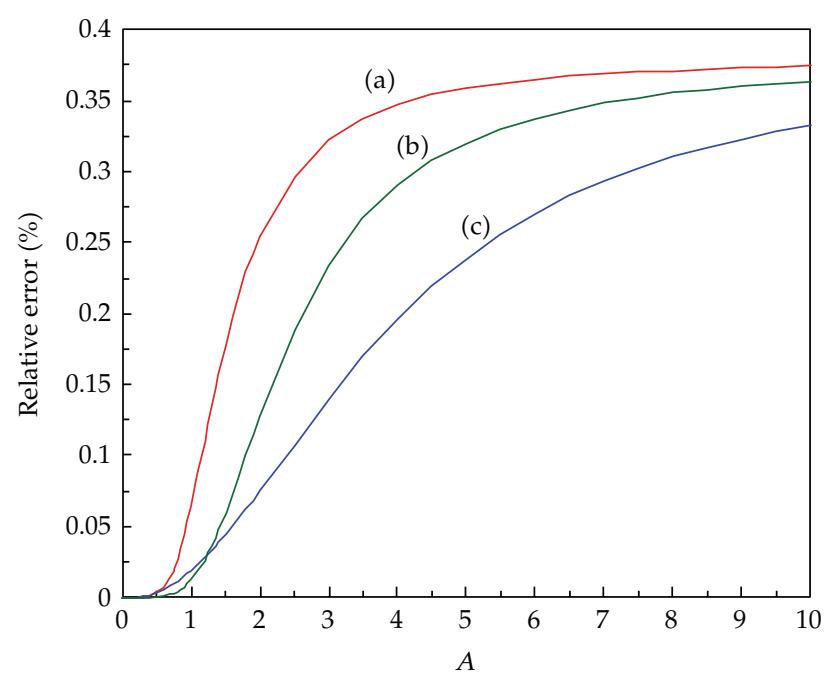

Figure 2: Comparison of approximate frequencies with exact frequencies for the cubic-quintic oscillator for (a) $\lambda_{1}=1, \lambda_{3}=1, \lambda_{5}=1$ (red), (b) $\lambda_{1}=5, \lambda_{3}=3, \lambda_{5}=1$ (green), and (c) $\lambda_{1}=1, \lambda_{3}=10, \lambda_{5}=1$ (blue).

For $\lambda_{1} \neq 0$ it is possible to obtain the following expressions for very small values of $A$ :

$$
\begin{gathered}
\omega_{e}(A) \approx \sqrt{\lambda_{1}}+\frac{3}{8} \frac{\lambda_{3}}{\sqrt{\lambda_{1}}} A^{2}+\frac{80 \lambda_{1} \lambda_{5}-21 \lambda_{3}^{2}}{256 \lambda_{1}^{3 / 2}} A^{4}+\frac{81 \lambda_{3}^{3}-304 \lambda_{1} \lambda_{3} \lambda_{5}}{2048 \lambda_{1}^{5 / 2}} A^{6}+\cdots, \\
\omega_{a}^{\mathrm{agm}}(A) \approx \sqrt{\lambda_{1}}+\frac{3}{8} \frac{\lambda_{3}}{\sqrt{\lambda_{1}}} A^{2}+\frac{80 \lambda_{1} \lambda_{5}-21 \lambda_{3}^{2}}{256 \lambda_{1}^{3 / 2}} A^{4}+\frac{81 \lambda_{3}^{3}-300 \lambda_{1} \lambda_{3} \lambda_{5}}{2048 \lambda_{1}^{5 / 2}} A^{6}+\cdots .
\end{gathered}
$$

For $\lambda_{5} \neq 0$ it is possible to obtain the following expression for very large values of $\lambda_{5} A^{4}$ :

$$
\omega_{e}(A) \approx 0.746834 \sqrt{\lambda_{5}} A^{2}
$$

Based on (3.9) and (5.4), the approximation compared with respect to the exact frequency is

$$
\lim _{\lambda_{5} A^{4} \rightarrow \infty} \frac{\omega_{a}^{\mathrm{agm}}(A)}{\omega_{e}(A)}=1.0038
$$

As can be seen, the relative error of the analytical approximate frequency $\omega_{a}^{\mathrm{agm}}$ is $0.38 \%$ in the limit as $\lambda_{5} A^{4} \rightarrow \infty$, which implies that the method proposed is suitable for solving (2.1) with $\lambda_{5} A^{4}$ much larger than unity. In Figure 2 we have plotted the comparison between the approximate and exact frequencies for different values of parameters $\lambda_{1}, \lambda_{3}$, and $\lambda_{5}$. 
For this cubic-quintic Duffing oscillator, Lai et al. obtained the following limits applying the Newton-harmonic balance method (NHBM) [1]:

$$
\begin{aligned}
& \lim _{\lambda_{5} A^{4} \rightarrow \infty} \frac{\omega_{1}(A)}{\omega_{e}(A)}=1.05856, \\
& \lim _{\lambda_{5} A^{4} \rightarrow \infty} \frac{\omega_{2}(A)}{\omega_{e}(A)}=1.01078, \\
& \lim _{\lambda_{5} A^{4} \rightarrow \infty} \frac{\omega_{3}(A)}{\omega_{e}(A)}=1.0023,
\end{aligned}
$$

for the first-, second-, and third-order analytical approximations, respectively. As we can see, the approximate frequency obtained in this paper, combining a cubication procedure (based on Chebyshev polynomials expansion of the restoring force), the arithmetic geometric mean (for approximating the exact frequency of the cubic Duffing oscillator), and the second-order rational harmonic balance method, is more accurate than the first-order and second-order frequencies obtained using the NHBM and slightly less accurate $(0.38 \%$ versus $0.23 \%)$ than the third-order frequency obtained using the NHBM.

In Table 2 we have presented the percentage errors for comparison of the approximate frequency with exact frequency for $\lambda_{1}=\lambda_{3}=\lambda_{5}=1$. For these parameters (3.5) can be written as follows:

$$
\omega_{a}^{\mathrm{agm}}(A)=\frac{1}{4}\left[\left(1+\frac{1}{2} A^{2}+\frac{5}{2} A^{4}\right)^{1 / 4}+\left(1+A^{2}+\frac{15}{2} A^{4}\right)^{1 / 4}\right]^{2} .
$$

Taking into account (3.9), (4.2), and (4.13) it is easy to verify that the analytical approximate solution for the cubic-quintic Duffing oscillator has the following asymptotic expression for $\lambda_{5} A^{4} \rightarrow \infty:$

$$
y_{\lambda_{5} A^{2} \rightarrow \infty}(t)=\frac{0.74928 \cos \left(0.749670 \lambda_{5}^{1 / 2} A^{2} t\right)}{1-0.25072 \cos ^{2}\left(0.749670 \lambda_{5}^{1 / 2} A^{2} t\right)} .
$$

We analyze this example because this situation corresponds to the worst case, since it has the approximate frequency whose relative error is the greatest.

Approximate and exact solutions for a few examples of the cubic-quintic Duffing oscillators are presented in Figures 3, 4, 5, and 6 for one complete cycle. The exact periodic solutions $\left(y_{e}=x_{e} / A\right)$ have been obtained by numerically integrating (2.1), and the proposed approximate periodic solutions $(y=x / A)$ have been computed using (4.2). In these figures parameter $h$ is defined as

$$
h=\frac{\omega_{e} t}{2 \pi} .
$$




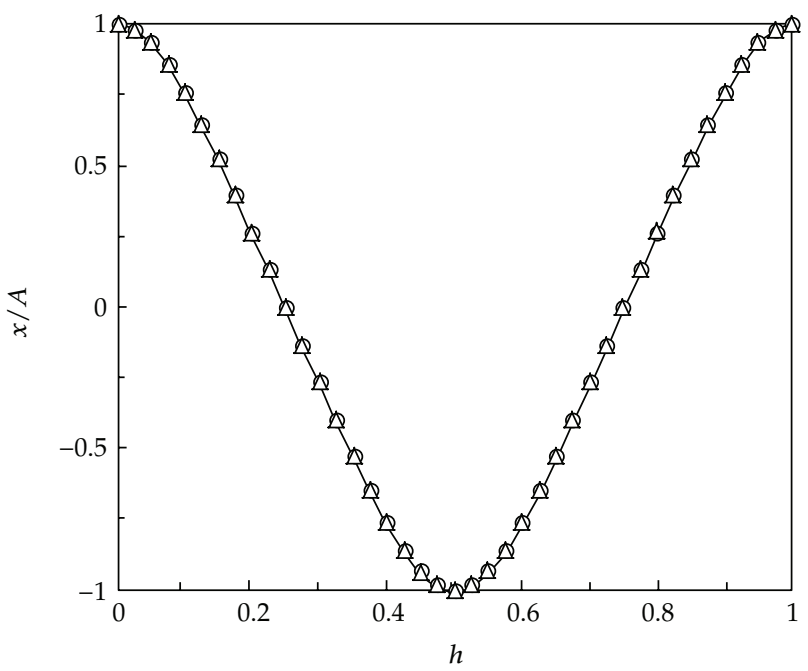

Figure 3: Comparison of the approximate solution (dashed line and triangles) with the numerical solution of (2.1) (continuous line and squares) for $\lambda_{1}=1, \lambda_{3}=10, \lambda_{5}=1$, and $A=1\left(L_{2}=0.00055478\right)$.

Table 2: Percentage errors for comparison of the approximate frequency with exact frequency for $\lambda_{1}=\lambda_{3}=$ $\lambda_{5}=1$.

\begin{tabular}{lccc}
\hline$A$ & $\omega_{a}^{\text {agm }}$ & $\omega_{e}$ & $(\%$ error $)$ \\
\hline 0.1 & 1.00377 & 1.00377 & 0.0000 \\
0.3 & 1.03554 & 1.03554 & 0.0000 \\
0.5 & 1.10657 & 1.10654 & 0.0027 \\
1 & 1.52456 & 1.52359 & 0.064 \\
3 & 7.29201 & 7.26863 & 0.32 \\
5 & 19.2503 & 19.1815 & 0.36 \\
8 & 48.4739 & 48.2946 & 0.37 \\
10 & 75.4588 & 75.1774 & 0.37 \\
20 & 300.355 & 299.223 & 0.38 \\
50 & 1874.66 & 1867.57 & 0.38 \\
70 & 3673.87 & 3659.98 & 0.38 \\
100 & 7497.18 & 7468.83 & 0.38 \\
300 & 67470.75 & 67215.57 & 0.38 \\
500 & 187418.88 & 186709.04 & 0.38 \\
700 & 367339.58 & 365949.25 & 0.38 \\
1000 & 749670.06 & 746834.69 & 0.38 \\
\hline
\end{tabular}

which tends to $0.746834 \lambda_{5}^{1 / 2} A^{2} t / 2 \pi$ as $\lambda_{5} A^{4} \rightarrow \infty$. The approximate solution, expressed as a function of $h$, which has been plotted in Figure 6 is

$$
y_{\lambda_{5} A^{2} \rightarrow \infty}(h)=\frac{0.74928 \cos (6.3070448 h)}{1-0.25072 \cos ^{2}(6.3070448 h)} .
$$




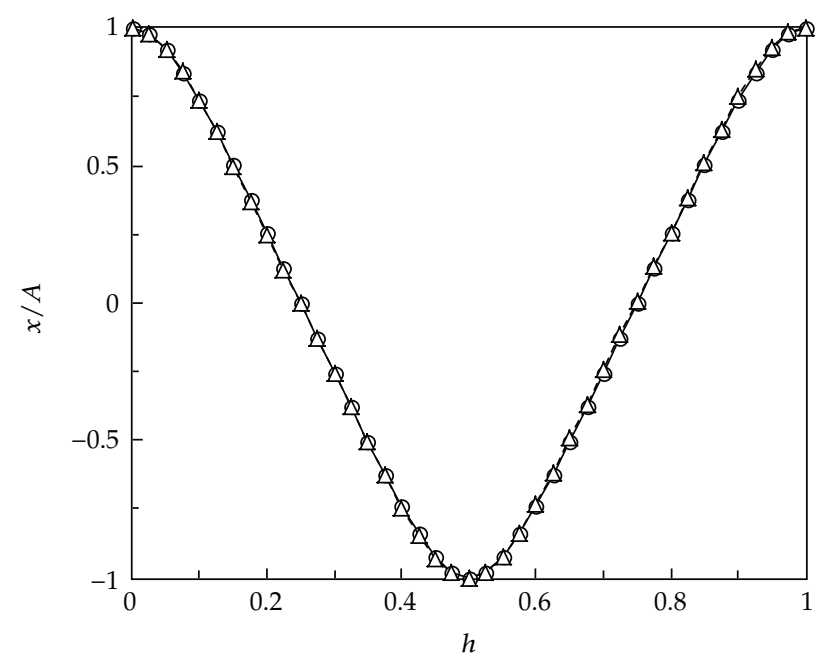

Figure 4: Comparison of the approximate solution (dashed line and triangles) with the numerical solution of (2.1) (continuous line and squares) for $\lambda_{1}=5, \lambda_{3}=3, \lambda_{5}=1$ and $A=3\left(L_{2}=0.0063554\right)$.

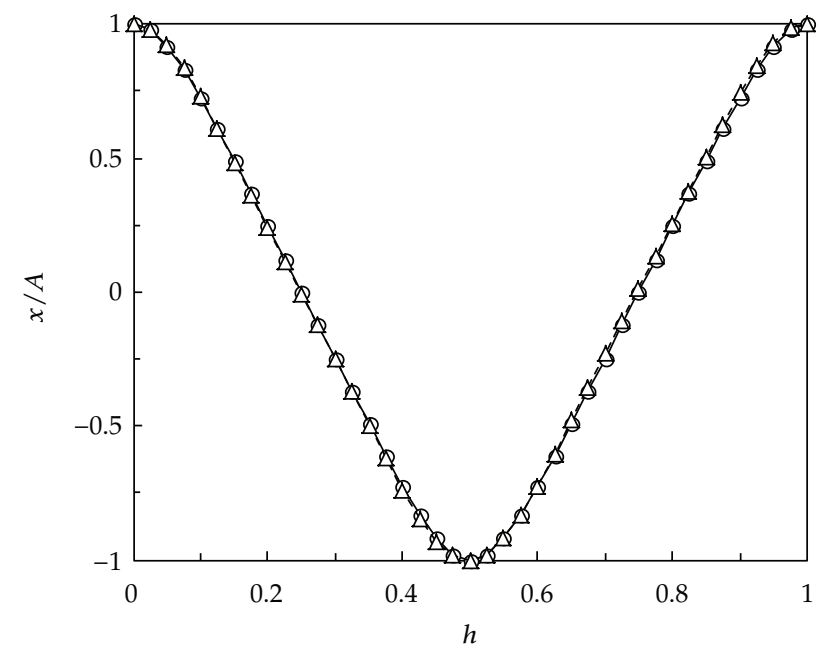

Figure 5: Comparison of the approximate solution (dashed line and triangles) with the numerical solution of (2.1) (continuous line and squares) for $\lambda_{1}=1, \lambda_{3}=1, \lambda_{5}=1$ and $A=3\left(L_{2}=0.0085975\right)$.

Parameters for oscillators analyzed in Figures 3-6 are presented in Table 3 in which the values for the $L_{2}$ norm errors have been also included in order to produce a global estimation of the accuracy of the approximate solutions [18]

$$
L_{2}=\sqrt{\int_{0}^{1}\left|y_{e}(h)-y(h)\right|^{2} d h} .
$$

The maximum value of $L_{2}$ norm is 0.0100931 and it is obtained for $\lambda_{5} A^{4} \rightarrow \infty$. In Figure 7 we have plotted the differences $\Delta=y_{e}-y$ for the oscillators presented in Figures 3-6. Figures 3-7 


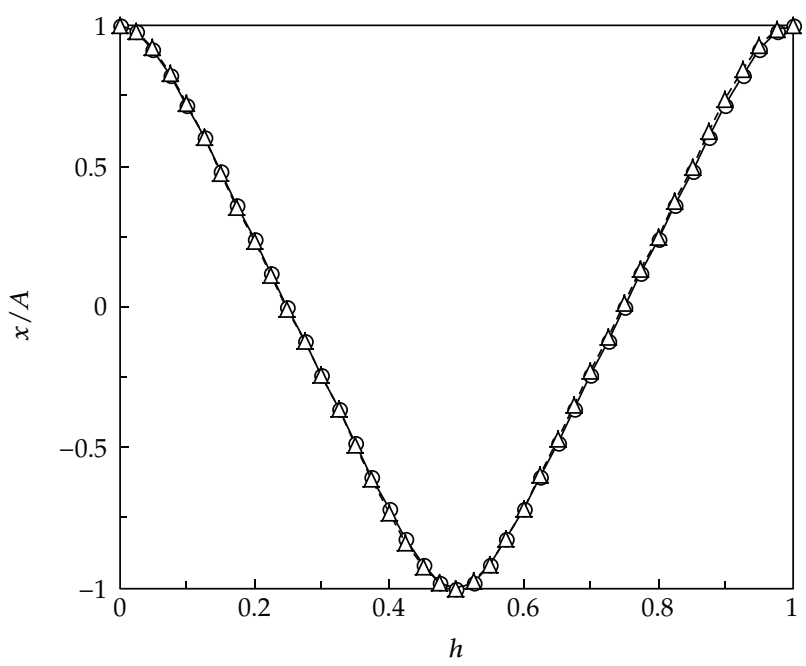

Figure 6: Comparison of the approximate solution (dashed line and triangles) with the numerical solution of (2.1) (continuous line and squares) for $\lambda_{5} A^{4} \rightarrow \infty\left(L_{2}=0.010093\right)$.

Table 3: Parameters for the cubic-quintic oscillators analyzed in Figures 3-6.

\begin{tabular}{cccccccc}
\hline$\lambda_{1}$ & $\lambda_{3}$ & $\lambda_{5}$ & $A$ & $\omega_{e}$ & $\omega_{a}^{\text {agm }}(\%$ error $)$ & $q$ & $L_{2}$ \\
\hline 1 & 10 & 1 & 1 & 2.96448 & $2.96504(0.019)$ & 0.15187 & 0.00055478 \\
5 & 3 & 1 & 3 & 8.37877 & $8.39842(0.23)$ & 0.20980 & 0.0063554 \\
1 & 1 & 1 & 3 & 7.26863 & $7.29201(0.32)$ & 0.23580 & 0.0085975 \\
& $\lambda_{5} A^{4} \rightarrow \infty$ & & $0.749670 \sqrt{\lambda_{5}} A^{2}$ & $0.746834 \sqrt{\lambda_{5}} A^{2}(0.38)$ & 0.25072 & 0.0100931 \\
\hline
\end{tabular}

show that (3.9) and (4.2) provide a good approximation to the exact periodic solution to the cubic-quintic Duffing oscillator.

\section{Conclusions}

Using the results obtained by applying a cubication method for the cubic-quintic Duffing oscillator, very simple and accurate expression for the frequency and the solution are obtained for the cubic-quintic Duffing oscillator. In this procedure the original nonlinear equation is replaced by a related "cubic Duffing equation" that approximates the original "cubic-quintic Duffing equation" closely enough to provide useful solutions. The cubic Duffing equation is exactly solved in terms of the complete elliptic integral of the first kind and the Jacobi elliptic function cn and these exact solutions are the approximate solutions to the cubicquintic Duffing equation. Using the relationship between the complete elliptical integral of the first kind and the arithmetic-geometric mean and the second-order rational harmonic balance method, closed-form expressions for the approximate frequency and the solution are obtained in terms of elementary functions with a relative error for the approximate frequency less than $0.38 \%$ for all values of the oscillation amplitude. The results of the method presented in this paper have been found to be in good agreement with those of the exact solutions obtained numerically. 


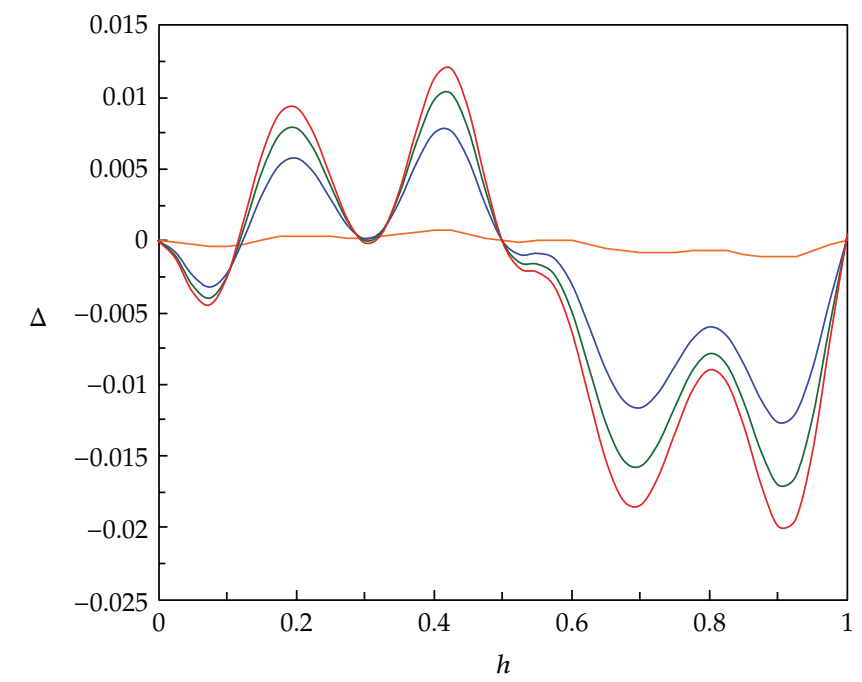

Figure 7: Difference between normalized numerical and approximate solutions for: (orange) $\lambda_{1}=1, \lambda_{3}=$ $10, \lambda_{5}=1$, and $A=1$, (blue) $\lambda_{1}=5, \lambda_{3}=3, \lambda_{5}=1$, and $A=3$; (green) $\lambda_{1}=1, \lambda_{3}=1, \lambda_{5}=1$, and $A=3$; (red) $\lambda_{5} A^{4} \rightarrow \infty$.

\section{Acknowledgments}

This work was supported by the "Generalitat Valenciana" of Spain, under Project PROMETEO/2011/021, and by the "Vicerrectorado de Tecnología e Innovación Educativa" of the University of Alicante, Spain, under Project GITE-09006-UA.

\section{References}

[1] S. K. Lai, C. W. Lim, B. S. Wu, C. Wang, Q. C. Zeng, and X. F. He, "Newton-harmonic balancing approach for accurate solutions to nonlinear cubic-quintic Duffing oscillators," Applied Mathematical Modelling. Simulation and Computation for Engineering and Environmental Systems, vol. 33, no. 2, pp. 852-866, 2009.

[2] J. I. Ramos, “On Linstedt-Poincaré technique for the quintic Duffing equation," Applied Mathematics and Computation, vol. 193, no. 2, pp. 303-310, 2007.

[3] C. W. Lim, R. Xu, S. K. Lai, Y. M. Yu, and Q. Yang, "Nonlinear free vibration of an elastically-restrained beam with a point mass via the Newton-harmonic balancing approach," International Journal of Nonlinear Sciences and Numerical Simulation, vol. 10, no. 5, pp. 661-674, 2009.

[4] A. Beléndez, G. Bernabeu, J. Francés, D. I. Méndez, and S. Marini, “An accurate closed-form approximate solution for the quintic Duffing oscillator equation," Mathematical and Computer Modelling, vol. 52, no. 3-4, pp. 637-641, 2010.

[5] J. H. Denman, “An approximate equivalent linearization technique for nonlinear oscillations," Journal of Applied Mechanics, vol. 36, no. 2, pp. 358-360, 1969.

[6] R. E. Jonckheere, "Determination of the period of nonlinear oscillations by means of Chebyshev polynomials," Zeitschrift fur Angewandte Mathematik und Mechanik, vol. 51, pp. 389-393, 1971.

[7] S. Bravo-Yuste, "Cubication of nonlinear oscillators using the principle of harmonic balance," International Journal of Non-Linear Mechanics, vol. 27, no. 3, pp. 347-356, 1992.

[8] S. Bravo-Yuste and A. Martín-Sánchez, "A weighted mean-square method of "cubication" for nonlinear oscillators," Journal of Sound and Vibration, vol. 134, no. 3, pp. 423-433, 1989.

[9] A. Beléndez, M. L. Álvarez, E. Fernández, and I. Pascual, “Cubication of conservative nonlinear oscillators," European Journal of Physics, vol. 30, no. 5, pp. 973-981, 2009. 
[10] A. Beléndez, D. I. Méndez, E. Fernández, S. Marini, and I. Pascual, “An explicit approximate solution to the Duffing-harmonic oscillator by a cubication method," Physics Letters A, vol. 373, pp. 2805-2809, 2009.

[11] A. Elías-Zúñiga, O. Martínez-Romero, and R. Córdoba-Díaz, "Approximate solution for the Duffingharmonic oscillator by the enhanced cubication method," Mathematical Problems in Engineering, vol. 2012, Article ID 618750, 12 pages, 2012.

[12] J. Cai, X. Wu, and Y. P. Li, "An equivalent nonlinearization method for strongly nonlinear oscillations," Mechanics Research Communications, vol. 32, no. 5, pp. 553-560, 2005.

[13] S. Bullett and J. Stark, "Renormalizing the simple pendulum," SIAM Review, vol. 35, no. 4, pp. 631640, 1993.

[14] A. Beléndez, E. Arribas, A. Márquez, M. Ortuño, and S. Gallego, “Approximate expressions for the period of a simple pendulum using a Taylor series expansion," European Journal of Physics, vol. 32, no. 5, pp. 1303-1310, 2011.

[15] R. E. Mickens, Oscillations in Planar Dynamic Systems, World Scientific, Singapore, Singapore, 1996.

[16] E. W. Weisstein, "Arithmetic-Geometric Mean," From MathWorld-A Wolfram Web Resource, http:/ / mathworld.wolfram.com/Arithmetic-GeometricMean.html .

[17] C. G. Carvalhaes and P. Suppes, "Approximation for the period of the simple pendulum based on the arithmetic-geometric mean," American Journal of Physics, vol. 76, no. 12, pp. 1150-1154, 2008.

[18] M. Febbo, "A finite extensibility nonlinear oscillator," Applied Mathematics and Computation, vol. 217, no. 14, pp. 6464-6475, 2011. 


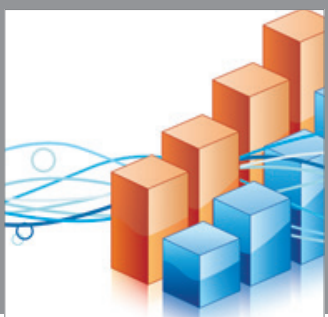

Advances in

Operations Research

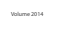

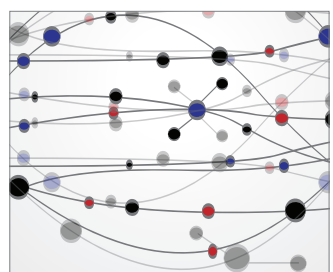

\section{The Scientific} World Journal
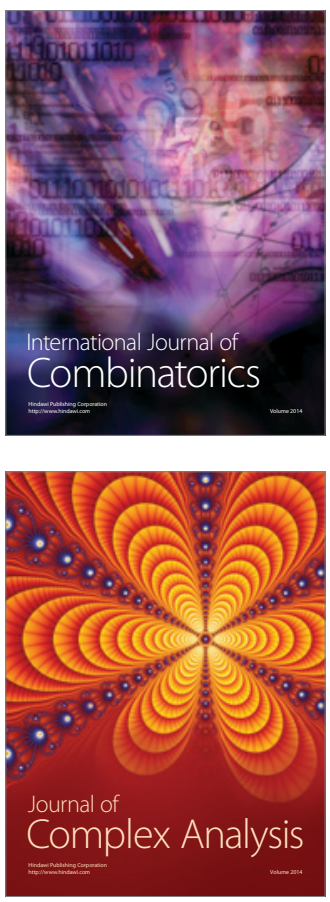

International Journal of

Mathematics and

Mathematical

Sciences
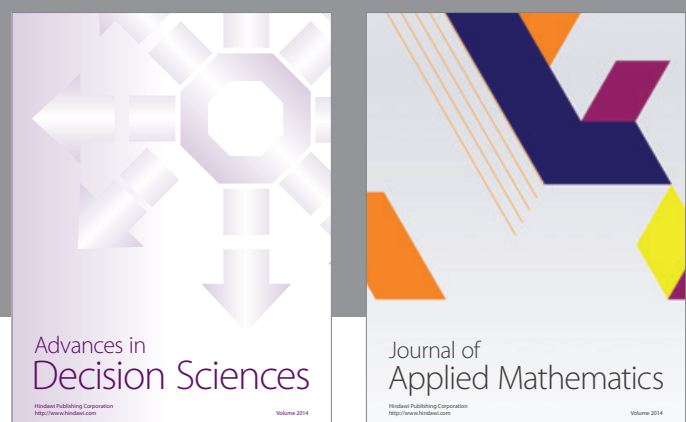

Journal of

Applied Mathematics
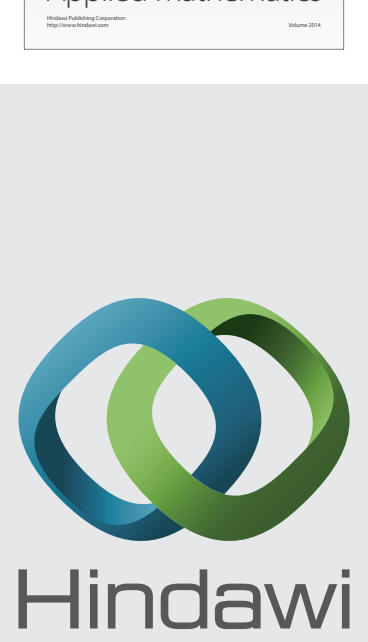

Submit your manuscripts at http://www.hindawi.com
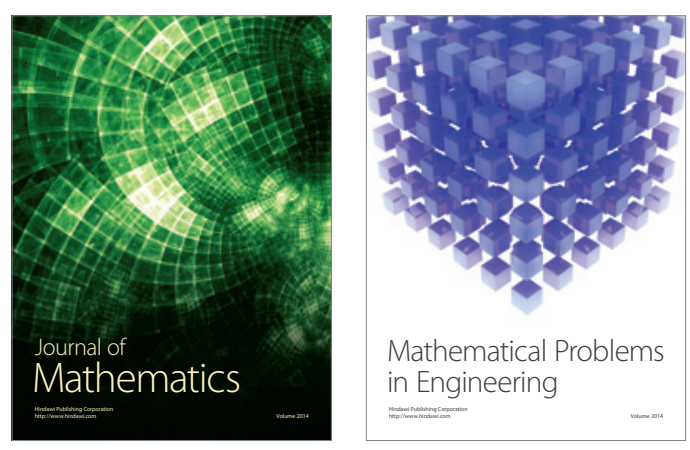

Mathematical Problems in Engineering
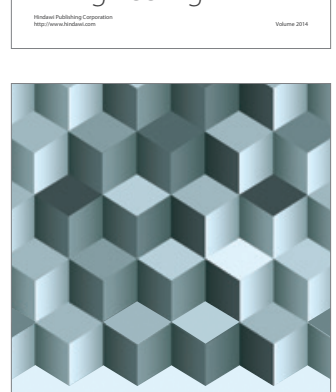

Journal of

Function Spaces
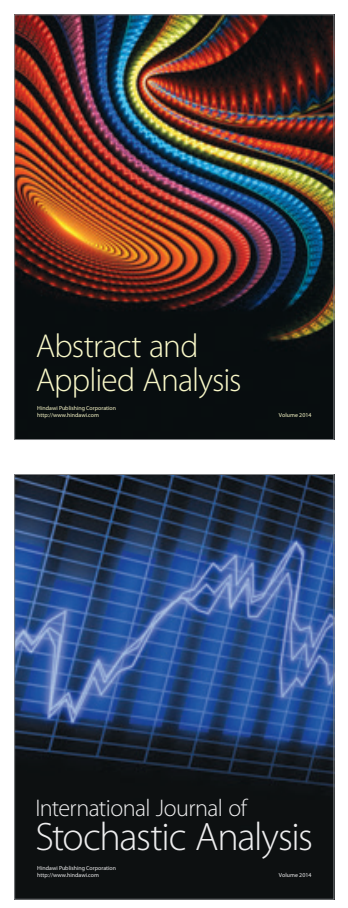

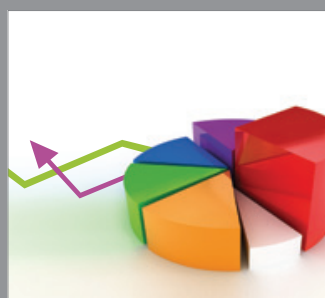

ournal of

Probability and Statistics

Promensencen
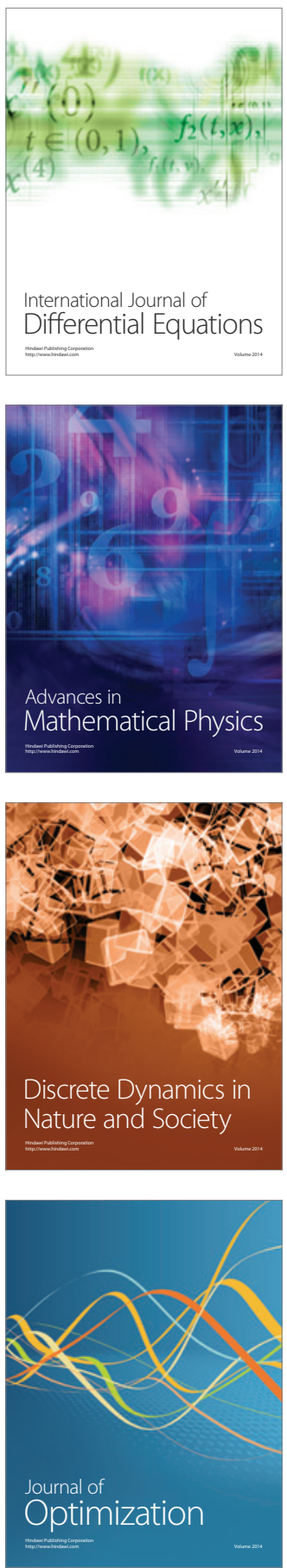